\title{
Frugivoria de larvas de Neosilba McAlpine (Diptera, Lonchaeidae) sobre Psittacanthus plagiophyllus Eichler (Santalales, Loranthaceae) no sudoeste de Mato Grosso do Sul, Brasil
}

\author{
Claudenir S. Caires ${ }^{1}$, Manoel A. Uchôa-Fernandes ${ }^{1}$, Jose Nicácio ${ }^{1} \&$ Pedro C. Strikis ${ }^{2}$
}

${ }^{1}$ Laboratório de Insetos Frugívoros, Faculdade de Ciências Biológicas e Ambientais, Universidade Federal da Grande Dourados, Caixa Postal 241, 79804-970 Dourados-MS, Brasil. uchoa.fernandes@ufgd.edu.br

${ }^{2}$ Departamento de Parasitologia, Instituto de Biologia, Universidade Estadual de Campinas, Cidade Universitária Prof. Zeferino Vaz, Barão Geraldo, 13083-970 Campinas-SP, Brasil.strikis@uol.com.br

\begin{abstract}
Larval frugivory of Neosilba McAlpine (Diptera, Lonchaeidae) on Psittacanthus plagiophyllus Eichler (Santalales, Loranthaceae) in southwestern Mato Grosso do Sul State, Brazil. Loranthaceae are hemiparasite plants with worldwide distribuition, represented in Brazil by six genera. The most important are Phthirusa, Psittacanthus and Struthanthus that parasitize a great diversity of host plants. This paper evaluated the occurrence and the seasonal fluctuation of flies infesting the fruits of Psittacanthus plagiophyllus in the municipalities of Anastácio, Aquidauana and Miranda, Mato Grosso do Sul, Brazil, where mistletoes are widespread. The samples were taken from June 1998 to July 2000 to obtain the hosts and its associated insects. All insects were reared until adult stage, and it were obtained 1,522 adults of Neosilba spp., of which 612 males were identified at species level: Neosilba bifida Strikis \& Prado (6 specimens), N. certa (Walker) (26 specimens), N. pendula (Bezzi) (16 specimens), N. zadolicha McAlpine \& Steyskal (4 specimens) and two different species, morphotype MSP1 (478 specimens) and morphotype 4 ( 82 specimens). The period of highest infestation by Neosilba spp. occurred during August 1998 and 1999, and morphotype MSP1 was significantly more abundant than all other species. The species morphotype 4 was the second most abundant, differing significantly from Neosilba zadolicha. Neosilba was the only genus of frugivorous fly infesting fruits of P. plagiophyllus and behaves as primary invader in this host.
\end{abstract}

KEYWORDS. Cerrado; hosts; fruit fly infestation; mistletoes.

RESUMO. Frugivoria de larvas de Neosilba McAlpine (Diptera, Lonchaeidae) sobre Psittacanthus plagiophyllus Eichler (Santalales, Loranthaceae) no sudoeste de Mato Grosso do Sul, Brasil. Loranthaceae são plantas hemiparasitas com distribuição geográfica mundial e representadas no Brasil por seis gêneros. Os mais importantes são Phthirusa, Psittacanthus e Struthanthus, os quais parasitam uma grande diversidade de plantas hospedeiras. Este trabalho avaliou a occurrência e a flutuação sazonal de moscas infestando os frutos de Psittacanthus plagiophyllus nos municípios de Anastácio, Aquidauana e Miranda, Mato Grosso do Sul, Brasil, onde ervas-de-passarinho são disseminadas. As coletas foram realizadas de junho de 1998 a julho de 2000 para obtenção de frutos maduros de $P$. plagiophyllus, seus insetos associados e catalogação dos seus hospedeiros. Os insetos foram criados para identificação, sendo obtidos 1.522 adultos de Neosilba spp., destes, 612 machos foram identificados a nível específico: Neosilba bifida Strikis \& Prado (6 indivíduos), N. certa (Walker) (26 indivíduos), N. pendula (Bezzi) (16 indivíduos), N. zadolicha McAlpine \& Steyskal (4 indivíduos) e duas diferentes espécies: morfotipo MSP1 (478 indivíduos) e morfotipo 4 (82 indivíduos). O período de mais alta infestação por Neosilba spp. ocorreu durante agosto de 1998 e de 1999 e, a espécie morfotipo MSP1 foi significativamente mais abundante que todas as outras. A morfotipo 4 foi a segunda mais abundante, diferindo significativamente de Neosilba zadolicha. Neosilba foi o único gênero de moscas frugívoras (Tephritoidea) infestante dos frutos de P. plagiophyllus e comportou-se como um invasor primário neste hospedeiro.

PALAVRAS-CHAVE. Cerrado; erva-de-passarinho; hospedeiros; infestação por moscas-das-frutas.

Loranthaceae são plantas hemiparasitas clorofiladas de caule nodoso formado por nós entumecidos, folhas simples, carnosas, verdes, em geral opostas, às vezes verticiladas ou alternas e, raramente áfilas. As flores são agrupadas em densas inflorescências (racemos, cimeiras e espigas), hermafroditas ou unissexuais, de cor amarela, amarelo-alaranjada, verde-oliva e até vermelhas (e.g. Psittacanthus calyculatus (DC.) G. Don (Gemtchújnicov 1976; Joly 1985; Pott \& Pott 1994). O fruto é ovóide, do tipo baga, pequeno em Oryctanthus, Oryctina, Phthirusa e Struthanthus e médio em Psittacanthus. A dispersão dos frutos de ervas-de-passarinho possui uma alta correlação com seus pássaros dispersores, influenciando naquela, o aspecto de controle da população, como também, na composição florística de uma dada região (Haigh 1996; Martinez Del Rio et al. 1996; Rizzini 1995).

A semente, após passar pelo trato digestório das aves, é eliminada com as fezes e ao cair sobre a casca de alguma árvore, geralmente, germina. O embrião apresenta dois, quatro, seis ou mais cotilédones (Gemtchújnicov 1976) e ao germinar, emite uma radícula que se transforma em haustório e penetra na casca da árvore hospedeira, alcançando o câmbio, onde exerce a função de raíz para retirar seiva bruta da planta hospedeira. 
As espécies de Loranthaceae são pragas de pomares e também atacam árvores e arbustos ornamentais, além de parasitarem árvores nativas. A infestação intensa associada às frutíferas causa a perda de qualidade, baixa produção de frutos e a morte da planta e a única forma de controle, sem grandes prejuízos ao hospedeiro, é através da sua poda (Johnson 1974 apud Venturelli 1981).

A frugivoria larval em frutos praticada por alguns grupos de dípteros (e.g. Tephritoidea, Lonchaeidae e Tephritidae) é altamente significativa na redução de sua qualidade, impedindo o seu comércio (Zucchi et al. 2004) e tais dípteros durante o período de entressafra podem se utilizar de outras plantas como hospedeiros alternativos.

Dentre as larvas frugívoras de moscas Tephritoidea destacam-se as espécies dos gêneros Anastrepha, Bactrocera, Rhagoletis e Ceratitis (Tephritidae) (Zucchi 2000), e as espécies dos gêneros Dasiops e Neosilba (Lonchaeidae), os quais ocorrem no Brasil, ambas com ampla distribuição geográfica (Uchôa-Fernandes \& Zucchi 2000).

As 16 espécies de Neosilba, com ampla distribuição geográfica, são consideradas as mais importantes dentre os insetos frugívoros, pois infestam enorme gama de frutos (Strikis \& Prado 2005). No Brasil existem 11 espécies de Neosilba, distribuídas pelos estados de (SP, MS BA, AP, AM, RJ, ES, PR, RS, SC, GO, ) (Aguiar-Menezes et al. 2007; Araújo \& Zucchi 2002; Bittencourt et al. 2006; Lourenção et al. 1996; Raga et al. 2005; Santos et al. 2004; Silva et al. 2006; Strikis et al. dados não publicados). Em Mato Grosso do Sul são relatados até o presente $N$. bifida Strikis \& Prado, N. certa (Walker), N. glaberrima (Wiedemann), N. pendula (Bezzi), N. zadolicha McAlpine \& Steyskal e morfotipo MSP1 e morfotipo 4 (Nicácio \& Uchôa-Fernandes, dados não publicados).

Diversos estudos de infestação de larvas frugívoras têm sido realizados com o intuito de identificar os diversos hospedeiros dessas moscas e seus inimigos naturais (Hymenoptera), a fim de conhecer sua biologia e gerar métodos de manejo populacional, principalmente para as espécies pragas em fruticulturas (Malavasi \& Zucchi 2000; Vilela et al. 2001).

Dentre os inimigos naturais das larvas frugívoras, existem os Braconidae (Hymenoptera, Opiinae), representados por espécies de Doryctobracon, Opius e Utetes (Campos 2001) e os Figitidae (Hymenoptera, Eucoilinae), por espécies de Aganaspis, Discerataspis, Lopheucoila, Odontosema e Trybliographa (Guimarães et al. 1999).

Neste estudo, a escolha do gênero Psittacanthus dentre os seis gêneros brasileiros de Loranthaceae, justifica-se pelas dimensões de seus frutos $(1,5 \times 1 \mathrm{~cm})$ já que os demais gêneros apresentam frutos muito pequenos com baixa proteção a inimigos naturais e pouca quantidade de nutrientes. Psittacanthus é representado por cerca de 80 espécies neotropicais, distribuídas do México até Honduras, chegando ao sul da América do Sul, passando pela região do Caribe, sendo dez espécies de ocorrência nas regiões Norte, Leste, Centro-Oeste e marginal do Bioma Cerrado (Barboza 2000). Psittacanthus plagiophyllus Eichler, é encontrada nas regiões
Norte, Nordeste e Centro-Oeste do Brasil e região Amazônica de outros países (Barboza 2000; Eichler 1868; Rizzini 1978), em ambientes de Cerrado e Mata (Barboza 2000). Segundo Barboza (2000) no Bioma Cerrado a espécie foi encontrada nos estados de MT, GO, DF e MS, sendo sua escolha para este trabalho justificada pela sua maior ocorrência nas localidades estudadas.

Os objetivos deste trabalho foram identificar os principais insetos frugívoros associados aos frutos de P. plagiophyllus; analisar a infestação dos insetos frugívoros e sua sazonalidade durante o período de frutificação e associar as espécies de moscas frugívoras do gênero Neosilba com seus parasitóides.

\section{MATERIALE MÉTODOS}

Foram realizadas 33 coletas em pontos aleatórios, nos municípios de Anastácio (20² $29^{\prime} \mathrm{S} / 55^{\circ} 49^{\prime} \mathrm{W}, 170 \mathrm{~m}$ ), Aquidauana ( $20^{\circ} 30^{\prime} \mathrm{S} / 55^{\circ} 47^{\prime} \mathrm{W}, 173 \mathrm{~m}$ ) e Miranda ( $20^{\circ} 14^{\prime} \mathrm{S} /$ $56^{\circ} 22^{\prime}$ W, $126 \mathrm{~m}$ ), Mato Grosso do Sul, entre os meses de junho de 1998 a julho de 2000.

Foram visitados periodicamente 26 hospedeiros $(21 \mathrm{em}$ Aquidauana, três em Anastácio e dois em Miranda) para o acompanhamento da fenologia de frutificação e para as coletas de frutos maduros. Nestes pontos as coletas foram intesificadas para aumentar o número de amostras, favorecendo assim, a obtenção de maior abundância e riqueza de insetos infestantes. Os indivíduos de Psittacanthus plagiophyllus sobre os hospedeiros não foram contados.

Os frutos coletados nos hospedeiros marcados foram levados para o Laboratório de Controle Biológico de Insetos do Departamento de Biociências, Universidade Federal de Mato Grosso do Sul (UFMS), Campus de Aquidauana, contados, pesados e submetidos à metodologia de UchôaFernandes \& Zucchi (1999), para a obtenção de todos os insetos associados.

As larvas pré-pupárias (LPp) foram coletadas diariamente (matutino e vespertino), contadas, identificadas ao nível de família e criadas em potes transparentes de acrílico com areia estéril umedecida com água esterilizada. Após a emergência os adultos (moscas e parasitóides) eram sacrificados e conservados em álcool 70\% para posterior identificação.

Os machos de lonqueídeos foram identificados pelo quarto autor, seguindo-se a metodologia de McAlpine \& Steyskal (1982).

A análise estatística foi feita utilizando-se o programa Bio Estat 4.0 (Ayres et al. 2005), com base no número de índivíduos. Para verificar a significância entre as médias, utilizou-se o teste de Kruskal-Wallis, usando mais de duas varáveis, com o intuito de verificar a abundância entre as espécies. O teste de MannWhitney foi empregado para comparar a significância entre as médias das espécies obtidas dos frutos.

Os exemplares de P. plagiophyllus e seus hospedeiros foram identificados e depositados na Universidade Federal de Mato Grosso do Sul e Universidade de Brasília (UnB). As moscas foram depositadas no Museu da Universidade Estadual de Campinas (UNICAMP) e os parasitóides 


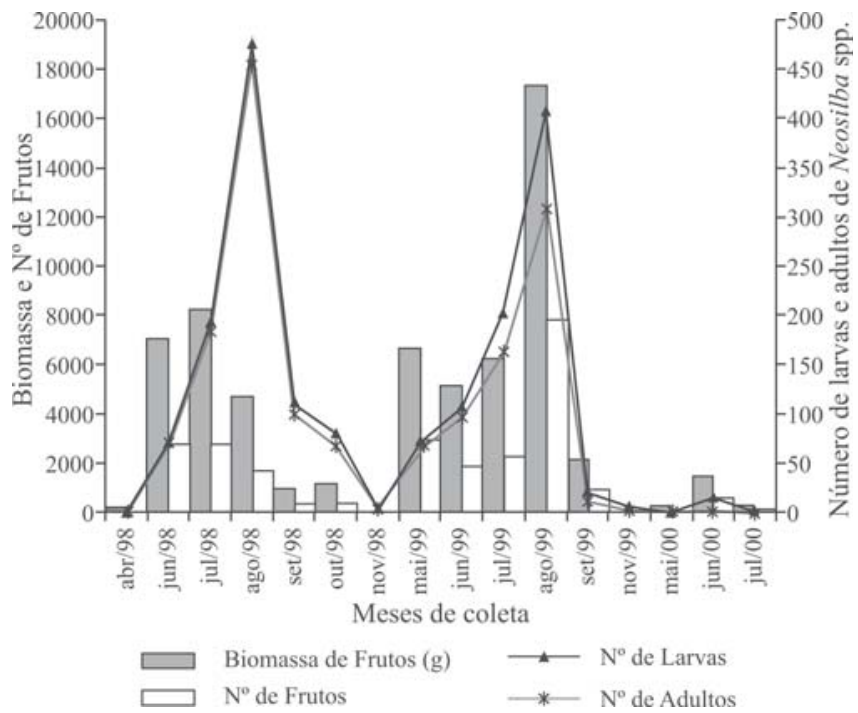

Fig. 1. Flutuação populacional de larvas e adultos de Neosilba spp. (Diptera, Lonchaeidae) sobre a frutificação de Psittacanthus plagiophyllus Eichler (Loranthaceae) no Pantanal sul-mato-grossense (Anastácio, Aquidauana e Miranda - junho de 1998 a julho de 2000).

(Hymenoptera) identificados e depositados na Coleção Zoológica do Departamento de Ecologia e Biologia Evolutiva, Universidade Federal de São Carlos - UFSCar.

\section{RESULTADOS}

A principal espécie de Psittacanthus obtida nas áreas amostradas foi P. plagiophyllus Eichler e a de menor ocorrência, $P$. calyculatus (DC.) G. Don, foi encontrada somente no município de Miranda, em apenas um dos locais marcados, infestando Psidium guajava L. (goiabeira). Devido à ausência e/ou baixa produção de frutos e larvas pré-pupárias (LPp), o indivíduo de $P$. calyculatus não foi incluído na contagem de frutos nem de larvas.

As dez famílias de plantas infestadas por $P$. plagiophyllus foram Anacardiaceae, Boraginaceae, Cecropiaceae, Combretaceae, Euphorbiaceae, Meliaceae, Fabaceae, Moraceae, Myrtaceae e Salicaceae (Tab. I). Os hospedeiros mais infestados foram Aroeira (Myracrodruon urundeuva (Engl.) Fr. Alb. - Anacardiaceae), Leiteira (Sapium longifolium (M. Arg.) Hule. - Euphorbiaceae), Angico (Anadenanthera colubrina var. cebil Bren. - Fabaceae), Timbaúva (Enterolobium contortisiliquum Vell. - Fabaceae), Goiabeira (Psidium guajava L. - Myrtaceae) e Chorão (Salix babylonica L. - Salicaceae) (Tab. I).

Quanto ao período de frutificação, P. plagiophyllus apresentou o início da produção de frutos no mês abril, com maturação iniciando-se no mês de maio com término em novembro (1998-2000) e pico de frutificação de junho a agosto de 1998 e 1999 (Fig. 1).

Foram coletados 24.710 frutos totalizando uma biomassa de $61,739 \mathrm{~kg}$. Destes frutos obteve-se um total de 1.764 larvas pré-pupárias (LPp) de Diptera, das quais emergiram 1.522 adultos de Neosilba spp. (Lonchaeidae) e 22 parasitóides, Utetes anastrephae Viereck (Hymenoptera, Braconidae) (Tab. I).

Dos 1.522 adultos emergidos, 252 foram danificados devido a sua longa permanência no conservante e 658 eram fêmeas, $o$ que impediu sua identificação. A abundância dos 612 indivíduos machos identificados foi a seguinte: Neosilba bifida Strikis \& Prado (6 indíviduos), N. certa (Walker) (26 indivíduos), N. pendula (Bezzi) (16 indivíduos), N. zadolicha McAlpine \& Steyskal (4 indivíduos) e as diferentes espécies morfotipo MSP1 (478 indivíduos) e morfotipo 4 (82 indivíduos).

O pico de infestação por larvas de Neosilba spp. ocorreu em agosto (1998/1999), com uma relação (LPp/fruto) de 0,28 (475 larvas/1.689 frutos) em 1998, e 0,05 (413 larvas/7840 frutos) em 1999 (Fig. 1).

Considerando-se as médias entre as quatro espécies de Neosilba e as duas morfoespécies (morfotipo MSP1 e morfotipo 4) e tomando-se como referência o número de machos, verificase uma maior abundância do morfotipo MSP1, a qual diferiu significativamente das demais espécies. O morfotipo 4 foi a segunda espécie mais abundante e diferiu significativamente de $N$. zadolicha, mas não apresentou diferença significativa com $N$. bifida, $N$. certa e $N$. pendula, as quais também não diferiram significativamente entre si (Tab. II).

\section{DISCUSSÃO}

A diversificação de hospedeiros das espécies de Loranthaceae é muito comum e seus gêneros são considerados grandes generalistas. P. plagiophyllus mostrou preferência pelas famílias Anacardiaceae e Fabaceae, o que também é observado para os gêneros Phthirusa (Loranthaceae) (Caires et al., dados não publicados) e Phoradendron (Viscaceae), que também são considerados generalistas (Caires \& Proença 2005).

Constatou-se que em Aquidauana, Anastácio e Miranda (Sudoeste de Mato Grosso do Sul), P. plagiophyllus é a espécie mais comum, no entanto $P$. calyculatus foi encontrada em Miranda e ausente nos demais municípios. Tal fato poderá ser explicado futuramente com a identificação e estudos de etologia dos agentes dispersores de sementes, já que os hospedeiros ocorreram em ambas as localidades.

A frutificação de $P$. plagiophyllus apresentou um período de entressafra de novembro a abril, no qual a planta se refaz da frutificação anterior, promovendo o crescimento e preparação para o próximo período de maturação dos frutos que inicia, geralmente, no mês de maio e se estende até novembro (Fig. 1), assemelhando-se à fenologia de $P$. calyculatus, que frutifica de julho a novembro (Barros et al. 2001).

A presença de Neosilba spp. como único grupo de Tephritoidea sobre os frutos de $P$. plagiophyllus, indica uma provável especificidade hospedeiro-frugívoro, pois, apesar da grande quantidade de frutos avaliados (24.710), este foi o único gênero de Diptera obtido. Apesar de Neosilba ter sido considerado como invasor secundário de frutos (Strikis \& Prado 2005), neste trabalho foi constatada uma associação 
Tabela I. Índice de infestação e viabilidade de larvas pré-pupárias (LPp) de Neosilba spp. (Diptera, Lonchaeidae) criadas de frutos de Psittacanthus plagiophyllus (Loranthaceae), amostrados no sudoeste de Mato Grosso do Sul ( junho/1998 a julho/2000).

\begin{tabular}{|c|c|c|c|c|c|c|c|}
\hline $\begin{array}{l}\text { Hospedeiros de P. plagiophyllus } \\
\text { e } \mathrm{N}^{\mathrm{o}} \text { de amostras (n) }\end{array}$ & $\begin{array}{l}\text { Qdade de } \\
\text { Frutos }\end{array}$ & $\begin{array}{l}\text { Biomassa } \\
(\mathrm{kg})\end{array}$ & $\begin{array}{l}\text { Qdade de } \\
\text { LPp }\end{array}$ & $\begin{array}{l}\text { Qdade de } \\
\text { Adultos }\end{array}$ & $\begin{array}{l}\text { Relação } \\
\text { LPp/Fruto }\end{array}$ & $\begin{array}{l}\text { Viabilidade } \\
\text { larval }(\%)\end{array}$ & $\begin{array}{l}\text { Insetos Associados } \\
\text { (número de indivíduos) }\end{array}$ \\
\hline \multicolumn{8}{|c|}{ 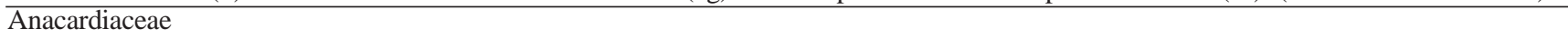 } \\
\hline Astronium graveoleus Jacq. (1) & 136 & 0,335 & 0 & 0 & 0,0 & & - \\
\hline Myracrodruon urundeuva (Engl.) Fr. Abl. (5) & 5.953 & 12,539 & 151 & 119 & 0,025 & 79 & $\begin{array}{l}\text { Neosilba sp. (danificado) } \\
3 \text { Utetes anastrephae } \\
\text { Morfotipo } 4 \text { (5) }\end{array}$ \\
\hline Tapirira guianensis Aubl. (1) & 140 & 0,342 & 67 & 67 & 0,471 & 100 & $\begin{array}{l}\text { Morfotipo MSP1 (34) } \\
\text { + Neosilba sp. (28) }\end{array}$ \\
\hline \multicolumn{8}{|l|}{ Boraginaceae } \\
\hline Cordia sp. (1) & 70 & 0,207 & 28 & 24 & 0,400 & 86 & $\begin{array}{l}\text { Morfotipo MSP1 (20) } \\
\text { Neosilba sp. (4) }\end{array}$ \\
\hline \multicolumn{8}{|l|}{ Cecropiaceae } \\
\hline Cecropia pachystachya Tréc. (2) & 511 & 1,471 & 108 & 85 & 0,211 & 79 & $\begin{array}{l}\text { Morfotipo MSP1 (29) } \\
\text { Morfotipo } 4 \text { (5) } \\
\text { N. certa (3) } \\
\text { + Neosilba } \mathrm{sp} . \text { (48) }\end{array}$ \\
\hline \multicolumn{8}{|l|}{ Combretaceae } \\
\hline Terminalia sp. (1) & 260 & 0,669 & 15 & 15 & 0,058 & 100 & $\begin{array}{l}\text { Morfotipo MSP1 (2) } \\
\text { Morfotipo } 4 \text { (2) } \\
\text { + Neosilba sp. (11) }\end{array}$ \\
\hline $\begin{array}{l}\text { Terminalia catappa L. (1) } \\
\text { Euphorbiaceae }\end{array}$ & \multicolumn{6}{|c|}{ Euphorbiaceae } & Neosilba sp. (danificado) \\
\hline Sapium longifolium (M. Arg.) Hule (17) & 11.508 & 31,497 & 1002 & 805 & 0,087 & 80 & $\begin{array}{l}\text { Morfotipo MSP1 (298) } \\
\text { Morfotipo } 4 \text { (60) } \\
\text { N. certa }(17) \\
\text { N. pendula }(10) \\
\text { N. zadolicha }(2) \\
\text { \& Neosilba } \text { sp. }(418) \\
\text { Neosilba } \text { sp. }(95 \\
\text { danificadas) } \\
8 \text { Utetes anastrephae }\end{array}$ \\
\hline \multicolumn{8}{|l|}{ Meliaceae } \\
\hline $\begin{array}{l}\text { Melia azedarach Blanco (1) } \\
\text { Fabaceae }\end{array}$ & 273 & 0,850 & 19 & \multicolumn{3}{|c|}{ Fabaceae } & Neosilba sp. (danificada) \\
\hline Acacia sp. (2) & 1707 & 3,686 & 60 & 44 & 0,035 & 73,34 & $\begin{array}{l}\text { Morfotipo MSP1 (4) } \\
\text { N. bifida (1) } \\
\text { N. pendula (2) } \\
\text { Neosilba } \text { sp. ( } 37 \\
\text { danificadas) }\end{array}$ \\
\hline Anadenanthera colubrina var. cebil Bren (4) & 1099 & 2,327 & 115 & 103 & 0,105 & 89,6 & $\begin{array}{l}\text { Morfotipo MSP1 (39) } \\
\text { Morfotipo } 4 \text { (1) } \\
\text { N. bifida (3) } \\
\text { N. certa (1) } \\
\text { N. pendula (3) } \\
\text { + Neosilba sp. (56) } \\
9 \text { Utetes anastrephae }\end{array}$ \\
\hline $\begin{array}{l}\text { Enterolobium contortisiliquum (Vell.) (5) } \\
\text { Moraceae }\end{array}$ & 911 & 2,002 & 14 & 8 & 0,015 & 57 & Neosilba sp. (danificada) \\
\hline Artocarpus integrifolia L. (2) & 66 & 0,188 & 31 & 30 & 0,470 & 97 & $\begin{array}{l}\text { Morfotipo MSP1 (10) } \\
\text { ㅇ Neosilba sp. }(20)\end{array}$ \\
\hline \multicolumn{8}{|l|}{ Myrtaceae } \\
\hline Psidium guajava L. (3) & 48 & 0,130 & 11 & 8 & 0,229 & 73 & $\begin{array}{l}\text { Morfotipo MSP1 (4) } \\
\text { + Neosilba sp. (4) }\end{array}$ \\
\hline \multicolumn{8}{|l|}{ Salicaceae } \\
\hline Salix babylonica L. (4) & 1676 & 4,692 & 143 & 126 & 0,085 & 88 & $\begin{array}{l}\text { Morfotipo MSP1 (38) } \\
\text { Morfotipo } 4 \text { (9) } \\
\text { N. bifida }(2) \\
\text { N. certa }(5) \\
\text { N. pendula }(1) \\
\text { N. zadolicha }(2) \\
\text { +Neosilba } \text { sp. }(69) \\
2 \text { Utetes anastrephae }\end{array}$ \\
\hline Total & 24.710 & & 1.765 & 1.454 & & 82,4 & \\
\hline
\end{tabular}


Tabela II. Estatística descritiva e comparações de médias de Neosilba spp. (Diptera) criadas de frutos de Psittacanthus plagiophyllus (Loranthaceae), amostrados no sudoeste de Mato Grosso do Sul (junho/ 1998 a julho/2000).

\begin{tabular}{lcccc}
\hline $\begin{array}{c}\text { Espécie de } \\
\text { moscas }\end{array}$ & Freqüência & Média & $\begin{array}{c}\text { Erro } \\
\text { Padrão }\end{array}$ & $\begin{array}{c}\text { Comparações de } \\
\text { Médias Mann- } \\
\text { Whitney }(\mathrm{p}<0,05)\end{array}$ \\
\hline Morfotipo MSP1 & 20 & 23.90 & 6.83 & $\mathrm{a}$ \\
Morfotipo 4 & 14 & 5.86 & 1.59 & $\mathrm{~b}$ \\
Neosilba certa & 8 & 3.25 & 1.11 & $\mathrm{bc}$ \\
N. pendula & 6 & 2.67 & 1.12 & $\mathrm{bc}$ \\
N. bifida & 5 & 1.20 & 0.20 & $\mathrm{bc}$ \\
N. zadolicha & 4 & 1 & 0 & $\mathrm{c}$
\end{tabular}

Kruskal-Wallis $(\mathrm{p}<0,05)$; nas comparações de médias as letras iguais não diferem significativamente entre si.

exclusivamente primária, pois, suas larvas representaram as únicas espécies de Diptera infestantes dos frutos de $P$. plagiophyllus. Dados referentes à invasão primária de Neosilba em frutos também foram relatados para Citrus sinensis e C. reticulata (Uchôa-Fernandes \& Zucchi 1999); em 22 hospedeiros silvestres ou cultivados nos cerrados de Mato Grosso do Sul (Uchôa-Fernandes et al. (2002) e em pomares comerciais (Araújo \& Zucchi (2002); Strikis \& Prado 2005).

Durante os dois anos de amostragem de larvas e adultos de Neosilba spp. nos frutos de P. plagiophyllus, obteve-se a mais alta infestação no mês de agosto (1998/1999), talvez devido a condições climáticas (Fig. 1). O número de frutos amostrados em agosto (1998) foi 1.689, com 475 larvas e 455 adultos, contrastando com os valores de agosto (1999), quando a quantidade de frutos aumentou (6.151 unidades) e o número de larvas e adultos diminuiu (62 e 130), respectivamente, mas o pico de infestação se manteve no mesmo período do ano anterior. Comparando-se a relação LPp/fruto de 1998 e 1999, percebe-se que a infestação no mês de agosto independe da quantidade e/ou qualidade dos frutos, mas provavelmente seja influenciada por fatores como temperatura, umidade e pluviosidade e, ainda mais importante, a provável ausência de outros frutos hospedeiros nessa época do ano.

Das seis diferentes espécies de Neosilba obtidas nesse trabalho, somente o morfotipo MSP1, morfotipo 4 e $N$. zadolicha apresentaram diferenças significativas entre si, sendo de maior destaque em número de indivíduos o morfotipo MSP1.

O único inimigo natural associado as espécies de Neosilba foi Utetes anastrephae (Braconidae), com 22 indivíduos, recuperados de larvas pré-pupárias, as quais conseguiram empupar.

Agradecimentos. Às Profas. M.Sc. Ubirazilda Maria Resende (UFMS), M.Sc. Maria Aglaene Barboza e Dra. Carolyn Elinore Barnes Proença (UnB) e Dra. Angélica Maria Penteado-Dias (UFSCAr) pelas identificações e ao CNPq pela concessão de bolsas de Iniciação Científica ao primeiro e ao terceiro autores.

\section{REFERÊNCIAS}

Aguiar-Menezes, E. L.; S. A. S. Souza; C. M. A. Santos; A. L. S. Resende; P. C. Strikis; J. R. Costa \& M. S. F. Ricci. 2007. Susceptibilidade de seis cultivares de Café Arábica às moscas-das-frutas (Diptera: Tephritoidea) em sistema orgânico com e sem arborização em Valença, RJ. Neotropical Entomology 36: 268-273.

Araújo, E. L. \& R. A. Zucchi. 2002. Hospedeiros e níveis de infestação de Neosilba pendula (Bezzi) (Diptera: Lonchaeidae) na região de Mossoró/Assu, RN. Arquivos do Instituto Biológico 69: 91-94.

Ayres, M.; M. Ayres Jr.; D. L. Ayres \& A. A. S. Santos. 2005. Bio Estat 4.0: aplicações estatísticas nas áreas das ciências Biológicas e Médicas. Belém-PA.

Barros, M. G.; V. Rico-Gray \& C. Díaz-Castelazo. 2001. Sincronia de floração entre Lantana camara L. (Verbenaceae) e Psittacanthus calyculatus (DC.) G. Don (Loranthaceae) ocorrentes em dunas de La Mancha, Veracruz, México. Acta Botanica Mexicana 57: 114.

Bittencourt, M. A. L.; A. C. M. Silva; Z. V. Bomfim; V. E. S. Silva; E. L. Araújo \& P. C. Strikis. 2006. Novos registros de espécies de Neosilba (Diptera: Lonchaeidae) na Bahia, Brazil. Neotropical Entomology 35: 282-283.

Caires, C. S. \& C. E. B. Proença. 2005. Viscaceae. Pp. 41-76. In: T. B. Cavalcanti \& A. E. Ramos (eds.). Flora do Distrito Federal, Brasil. Vol. 4. Embrapa Recursos Genéticos e Biotecnologia, Brasília.

Campos, D. F. 2001. Lista de los géneros de avispas parasitóides Braconidae (Hymenoptera: Ichneumonoidea) de la Región Neotropical. Biota Colombiana 2: 193-232.

Eichler, A. G. 1868. Loranthaceae, p. 1-135. In: C. F. P. Martius (eds.). Flora Brasiliensis 5, pt. 2.

Gemtchújnicov, I. D. 1976. Manual de taxonomia vegetal: plantas de interesse econômico. Ed. Agronômica Ceres, São Paulo. 368p.

Guimarães, J. A.; R. A. Zucchi; N. B. Diaz; M. F. Souza-Filho \& M. A Uchôa-Fernandes. 1999. Espécies de Eucoilinae (Hymenoptera: Cynipoidea: Figitidae) parasitóides de larvas frugívoras (Diptera: Tephritidae e Lonchaeidae) no Brasil. Anais da Sociedade Entomológica do Brasil 28: 263-273.

Haigh, S. L. 1996. Saltcedar (Tamarix ramosissima), an uncommon host for desert mistletoe (Phoradendron californicum). Great Basin Naturalist 56: 186-187.

Joly, A. B. 1985. Botânica: Introdução à taxonomia vegetal. $7^{\mathrm{a}}$ ed. São Paulo. Ed. Nacional, 777 p.

Lourenção, A. L.; J. O. Lorenzi \& G. M. B. Ambrosano. 1996. Comportamento de clones de mandioca em relação a infestação por Neosilba perezi (Romero \& Ruppell) (Diptera: Lonchaeidae). Scientia Agricola 53: 304-309.

Malavasi, A. \& R. A. Zucchi (eds). 2000. Moscas-das-frutas de Importância Econômica no Brasil: conhecimento básico e aplicado. Ribeirão Preto: Holos. 327 p.

Martinez del Rio, C.; A. Silva; R. Medel \& M. Hourdequin. 1996. Seed dispersers as disease vectors: bird transmission of mistletoe seeds to plant hosts. Ecology 77: 912-921.

McAlpine, J. F. \& G. C. Steyskal. 1982. A revision of Neosilba McAlpine with a key to the world genera of Lonchaeidae (Diptera). Canadian Entomologist 114: 105-137.

Pott, A. \& V. J. Pott. 1994. Plantas do Pantanal. Brasília, EMBRAPA, $320 \mathrm{p}$.

Raga, A.; R. A. Machado; M. F. Souza-Filho; M. E. Sato \& R. C. Siloto. 2005. Tephritoidea (Diptera) species from Myrtaceae fruits in the State of São Paulo, Brazil. Entomotropica 20: 11-14.

Rizzini, C.T. 1978. Loranthaceae. In: The Botany of the Guayana Highland, Part X. B. Maguire (Ed.). Memories of New York Botanical Garden 29: 23-36.

Rizzini, C. T. 1995. Flora da Serra do Cipó, Minas Gerais: Loranthaceae. Boletim do Instituto de Botânica 14: 207-221.

Santos, W. S.; C. A. L. Carvalho \& O. M. Marques. 2004. Registro de Neosilba zadolicha McAlpine \& Steyskal (Diptera: Lonchaeidae) em Umbu-Cajá (Anacardiaceae). Neotropical Entomology 33: 653-654. 
Silva, F. F.; R. N. Meirelles; L. R. Redaelli \& F. K. D. Soglio. 2006. Diversity of flies (Diptera: Tephritidae and Lonchaeidae) in organic citrus orchards in the Vale do Rio Caí, Rio Grande do Sul, Southern Brazil. Neotropical Entomology 35: 666-670.

Strikis, P. C. \& A. P. Prado. 2005. A new species of the genus Neosilba (Diptera: Lonchaeidae). Zootaxa 828: $1-4$.

Uchôa-Fernandes, M. A.; I. Oliveira; R. M. S. Molina \& R. A. Zucchi. 2002. Species diversity of frugivorous flies (Diptera: Tephritoidea) from hosts in the Cerrado of the State of Mato Grosso do Sul, Brazil. Neotropical Entomology 31: 515-524.

Uchôa-Fernandes, M. A. \& R. A. Zucchi. 1999. Metodología de colecta de Tephritidae y Lonchaeidae frugívoros (Diptera: Tephritoidea) y sus parasitoides (Hymenoptera). Anais da Sociedade Entomológica do Brasil 28: 601-610.

Uchôa-Fernandes, M. A. \& R. A. Zucchi. 2000. Mato Grosso e Mato
Grosso do Sul, p. 241-245. In: A. Malavasi, R. A. Zucchi (eds.). Moscas-das-frutas de importância econômica no Brasil: conhecimento básico e aplicado. Ribeirão Preto: Holos, 327p.

Venturelli, M. 1981. Estudos sobre Struthanthus vulgaris Mart.: anatomia do fruto e semente e aspectos de germinação, crescimento e desenvolvimento. Revista Brasileira de Botânica 4: 131147.

Vilela, E. F.; R. A. Zucchi \& F. Cantor (eds.). 2001. Histórico e impacto das pragas introduzidas no Brasil. Ribeirão Preto: Holos, 173 p.

Zucchi, R. A. 2000. Taxonomia, p. 13-24. In: A. Malavasi, R. A. Zucchi (eds.). Moscas-das-frutas de importância econômica no Brasil: conhecimento básico e aplicado. Ribeirão Preto: Holos. 327 p.

Zucchi, R. A.; A. Malavasi; A. S. Nascimento \& J. M. M. Walder. 2004. Prejuízos das moscas-das-frutas na exportação de citros. Visão Agrícola 2: 73-77. 